\title{
تحديد الظروف المثلى لأنتاج الكايتوسان من الفطر Aspergillus oryzae SU-B2 بطريقة تخمرات الحالة السائلة
}

\section{Optimization Condition of Chitosan Produced by Submerged Fermentation from Aspergillus oryzae SU-B2}

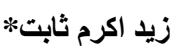

Aya Ahmed Abdulatoof
جامعة بغداد/ كلية الزبر اعة جبر

|جامعة النهرين مركز بحوث الثقانات الاحيائية

Hameed Abood Jaber

College of Agriculture/ Baghdad University

*Biotechnology Research Center/ AL-Nahrain University
آيها أحمد عبد اللطيف

Zaid A. Thabit*

E-mail:zaidakrm@yahoo.com

الكايتوسان الذي يمكن الحصول عليه بعد ازالة مجاميع الاستيل من الكايتين، هو سكر متعدد يتشكل اساسا من وحدات متعددة من الدي

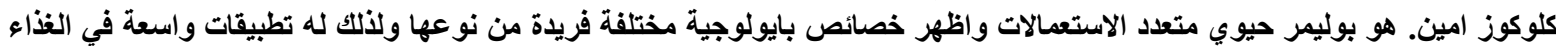

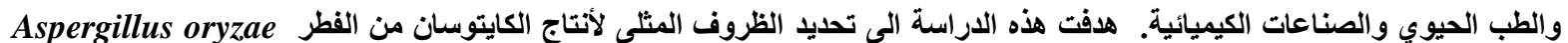
SU-B2

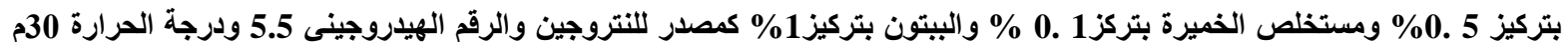

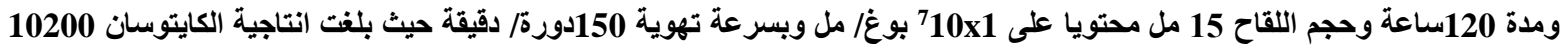

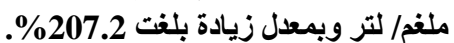

\section{Abstract}

Chitosan, the deacetylated form of chitin, is a polysaccharide formed primarily of repeating units of Dglucosamine. It is a versatile biopolymer exhibiting various unique biological properties hence finds wide applications in food, biomedical and chemical industries. This study was conducted to evaluate optimum condition for chitosan production from Aspergillus oryzae SU-B2. The result have showed the best concentration $2 \%$ glucose as carbon sources, while $0.5 \%\left(\mathrm{NH}_{4}\right)_{2} \mathrm{SO}_{4}$ and $0.1 \%$ yeast extract were optimum concentration as nitrogen sources. The optimum pH was 5.5 whereas the optimum temperature is $30^{\circ} \mathrm{C}$ with incubation time $120 \mathrm{~h}$ and agitation rate $150 \mathrm{rpm}$. The high yield of chitosan $10200 \mathrm{mg} / \mathrm{ml}$ was obtained with average increase $207.2 \%$.

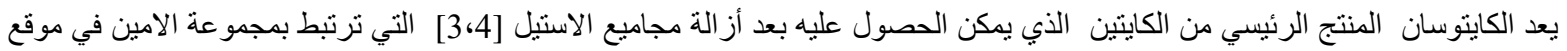

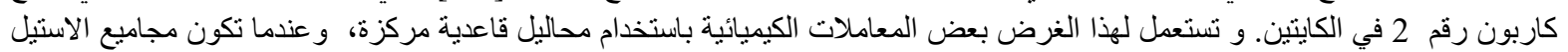

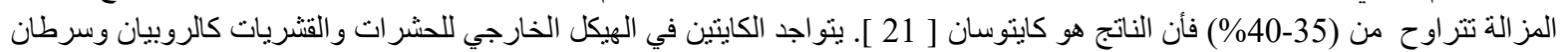

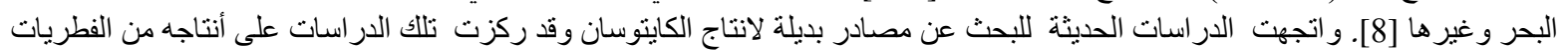

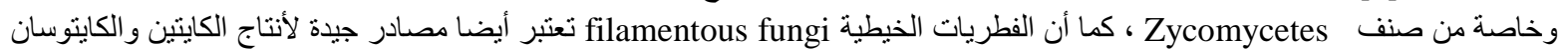

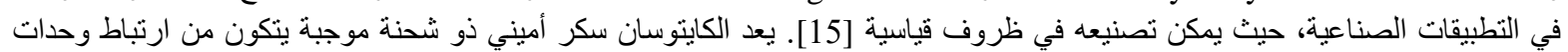

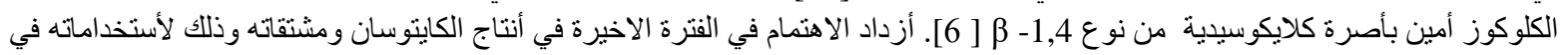

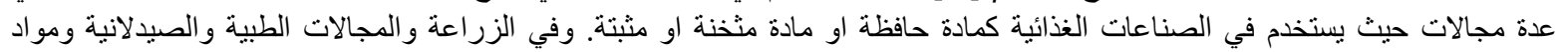

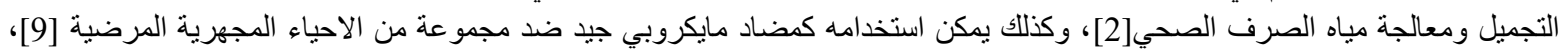

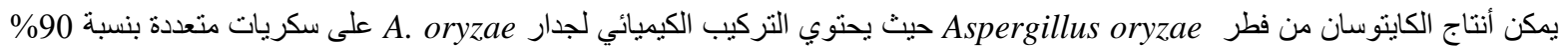

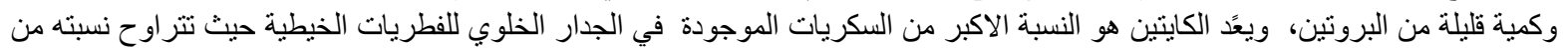

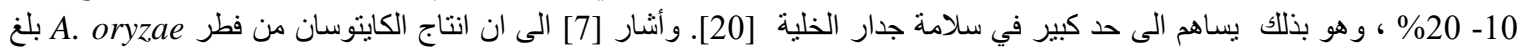

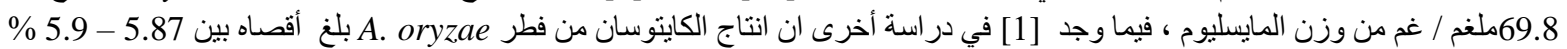

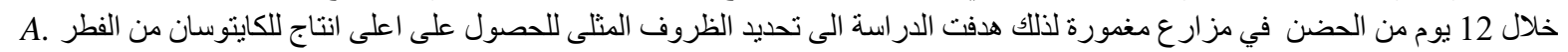

.oryzae

$$
\text { الاحياء المستخدمة وطئق العمل }
$$

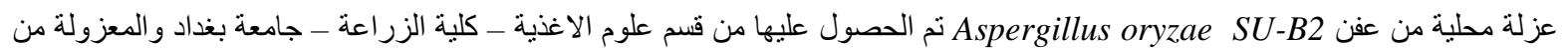

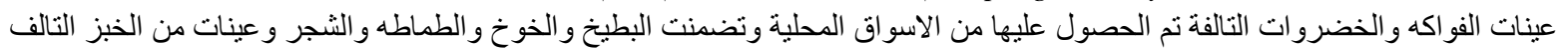
و اللبن و عينة من تربه مزيجية من حقول كلية الزر اعة /جامعة بغداد في الجادرية. 
وسط الانتاج ( YPG

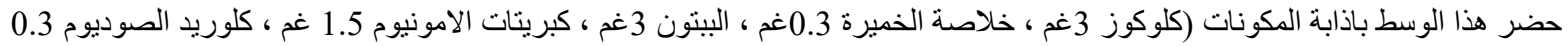

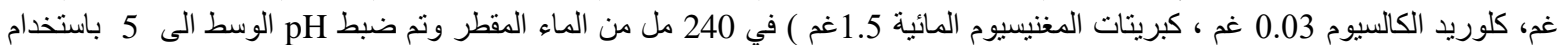

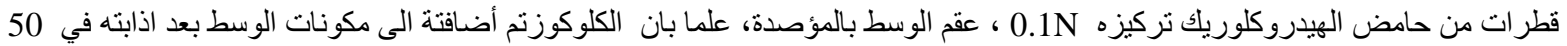

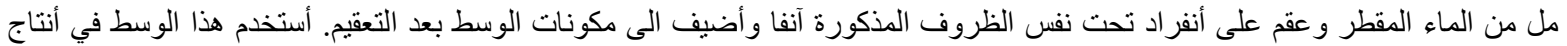

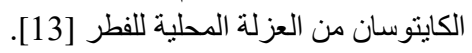
انتاج وأستخلاص الكايتوسنان التزان

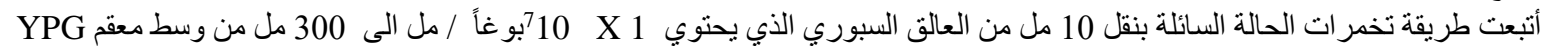

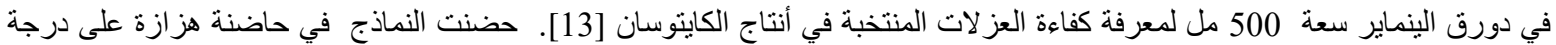
30م مدة 4 أيام في دوارق و بسر دوعة 150 دورة /دقيقة، أجري حصد الغزل الفطري بعد أنتهاء فترة الحضن بترشيح وسط الانتاج بقمع بخنّر

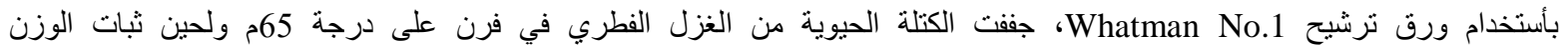

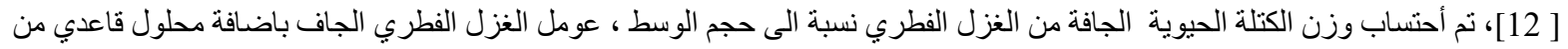

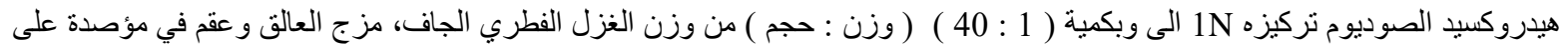

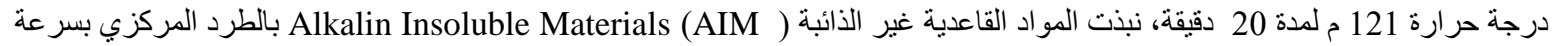

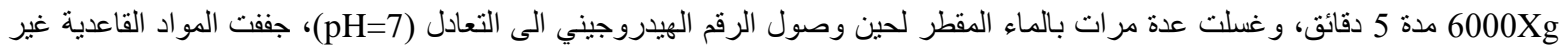

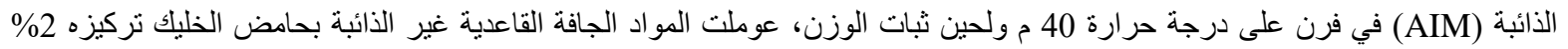

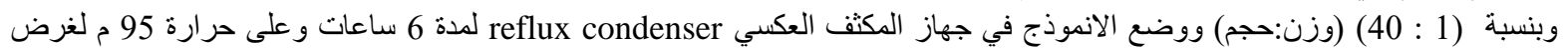

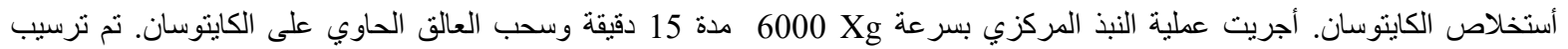

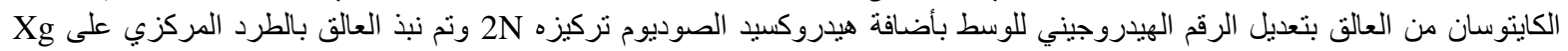

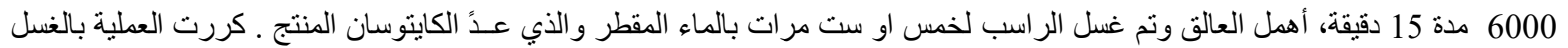

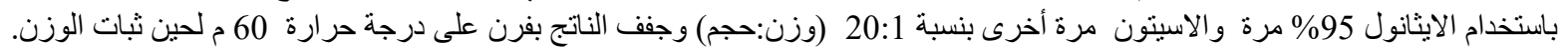

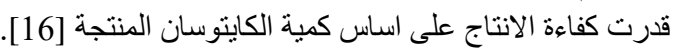
تحديد الظروف المثلى لأنتاج الكايتوسان 1- تحديد المصدر الكاربوني الامثل

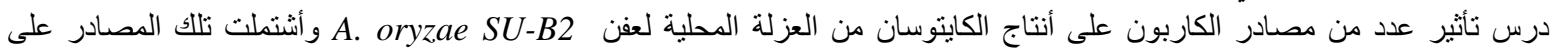

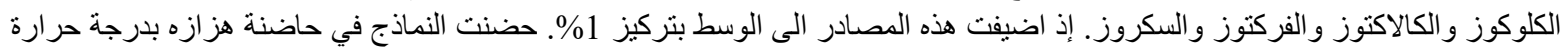

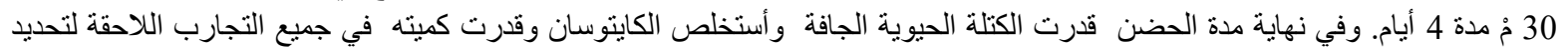

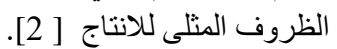
2- تحديد التركيز الامثل للمصدر الكاربوني لإني

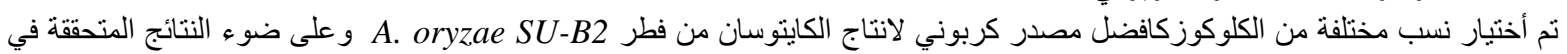

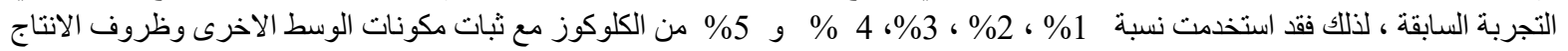

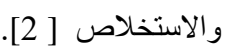

3- تحديد المصدر النتروجيني الامثل

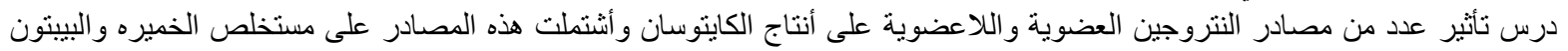

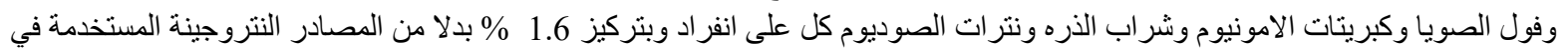

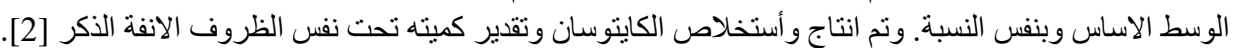

4- تحديد الرقم الهيدروجيني الامثل

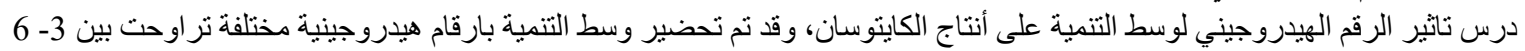

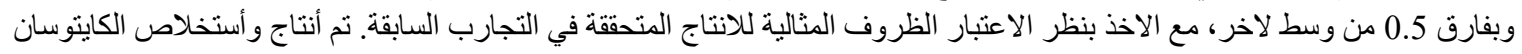

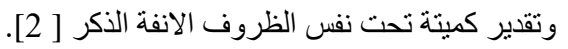
5- تحديد درجة الحرارة المثلى للأنتاج

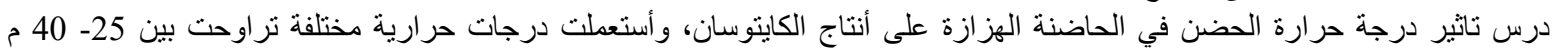

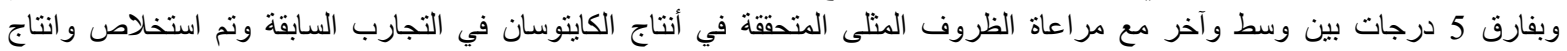

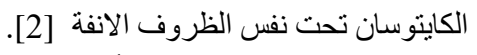
6- تحديد فترة الحضن المثلى للأنتاج

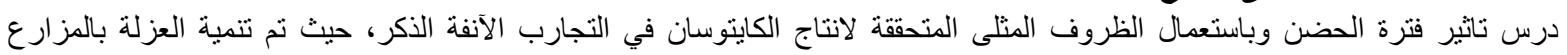

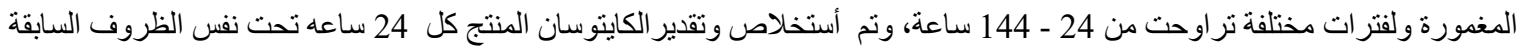

7- تحديد حجم اللقاح الامثل

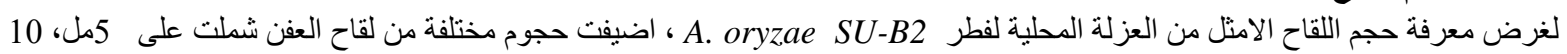

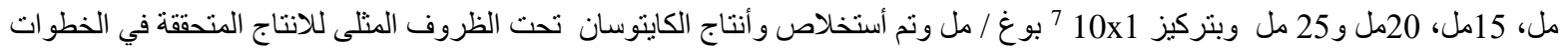

السابقة [2]، 
8- تحديد عدد الدورات المثلى للانتاج

درس تاثير سرعة التهوية خلال فترة الحضن على أنتاج الكايتوسان، حيث حضنت مزارع الفطر في حاضنة هزازة تحت الظروف المثالية

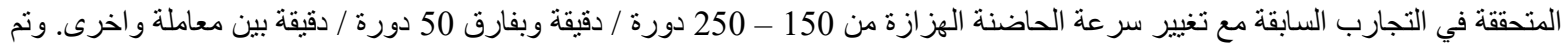

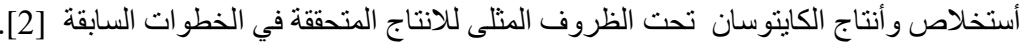

النتائج و المناقشة

تحديا المصدر الكاربوني الامثل

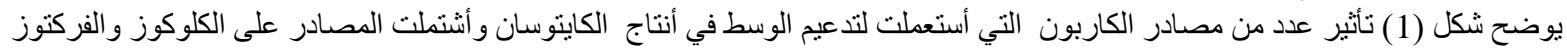

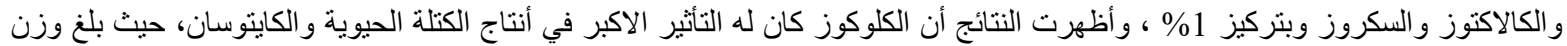

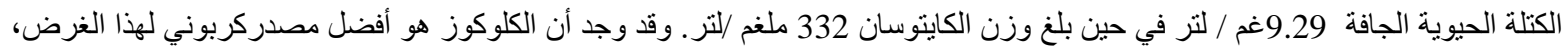

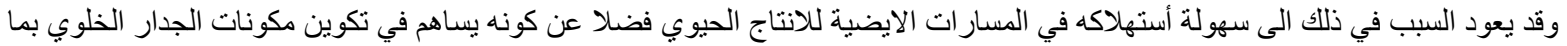

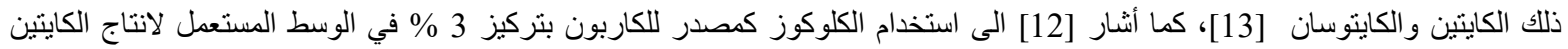

و الكايتو سان من عز لات مختلفة من عفن Penicillium spp.

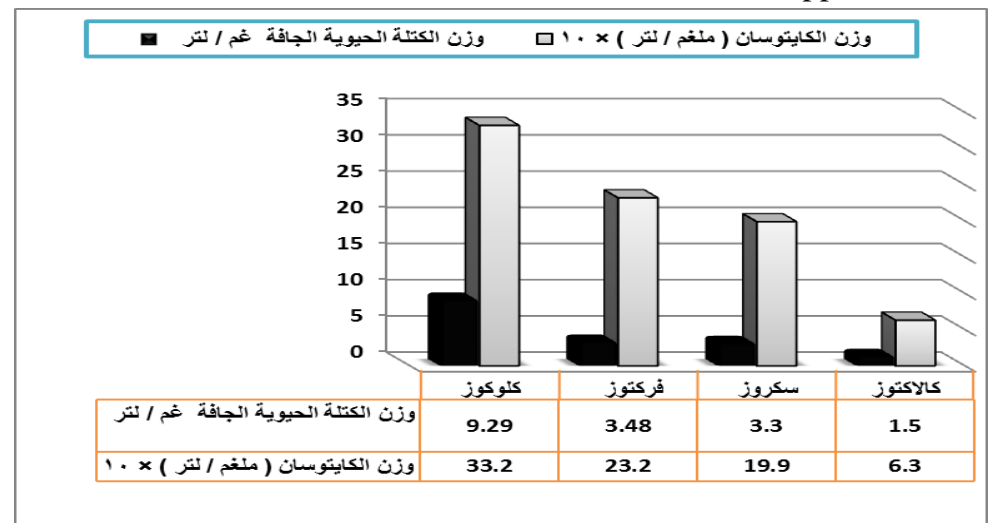

شكل(1): تأثير مصادر مختلفة من الكاريون على أنتاج الكايتوسان من عزلة محلية من فطر A. oryzae SU-B2.

تأثير تركيز الكلوكوز الامثل في الانتاج

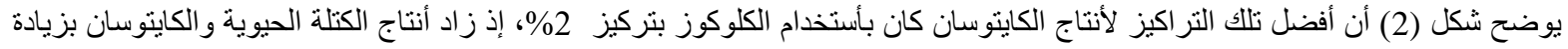

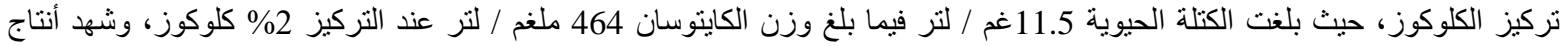

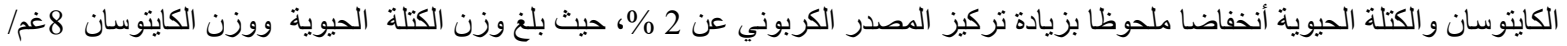

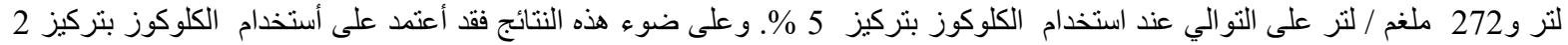

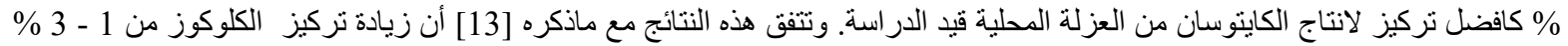

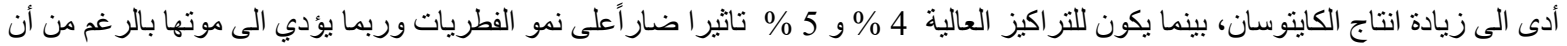

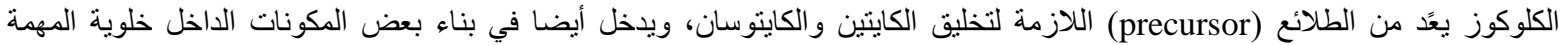

الاخرى في الخلية [ [5].

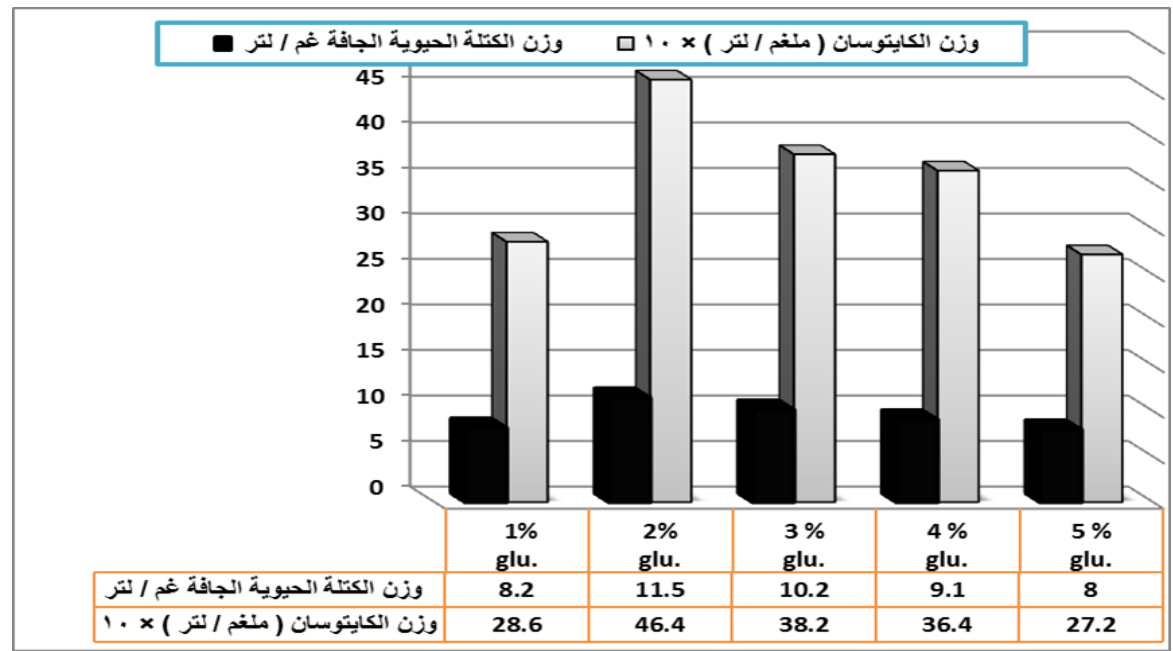

A. oryzae SU-B2 شكل (2): تاثير تركيز الكلوكوز في انتاج الكايتوسان من من عزلة محلية من فطر الكائرسان

تأثير المصدر النتروجيني على انتاج الكايتوسان

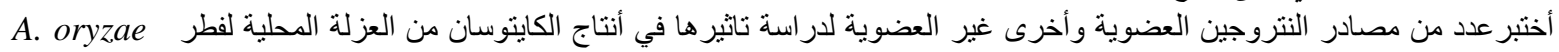
وشملت تللك المصادر التي استعملت لتدعيم وسط الانتاج على مستخلص الخميرة ( SU-B2 


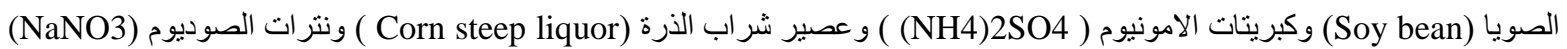

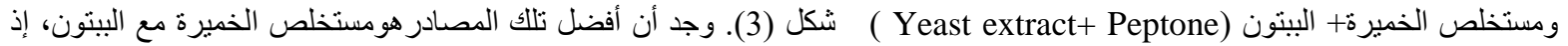

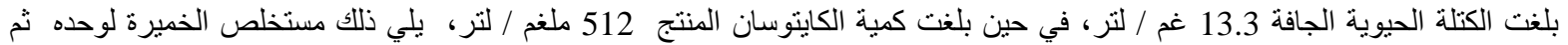

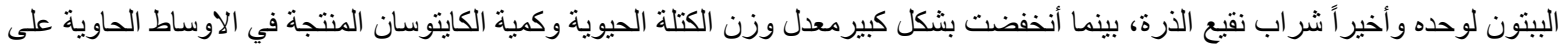
المصادر النتروجينية اللاعضوية، أما في حالة غياب المصدر النتروجيني فقد أنخفضت الحصيلة من الكايتوسان والكتلة الحيوية الى 90\% ، 96

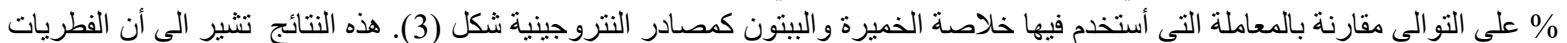

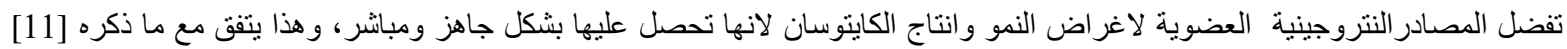

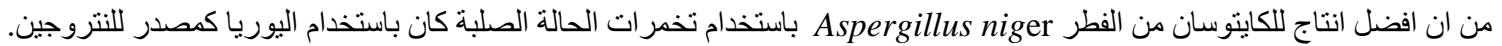

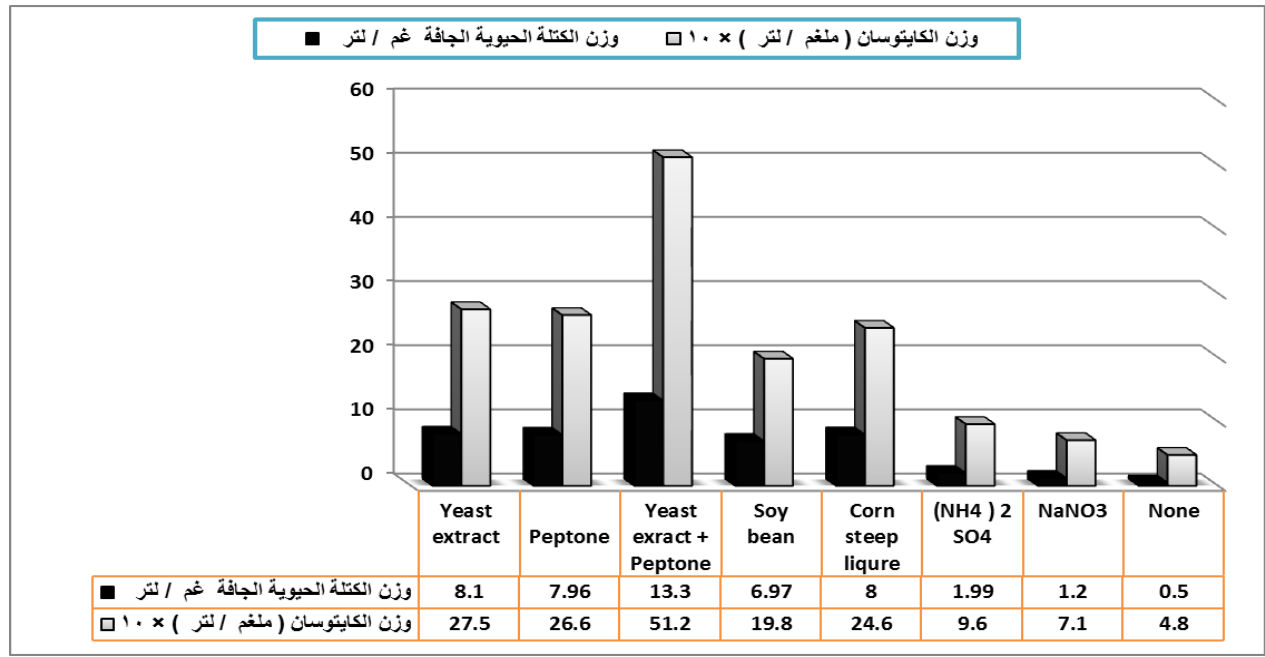

\section{شكل(3): تأثير مصدر النتروجين في انتاج الكايتوسان من من عزلة محلية من فطر A. oryzae SU-B2} الكلوكوز بتركيز 2 \% مصدرا للكاربون.

تحديد الرقم الهيدروجيني الامثل

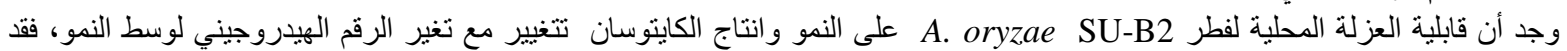

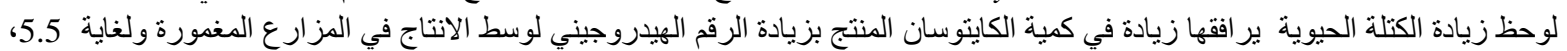

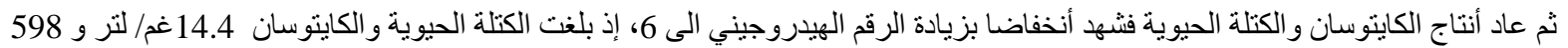

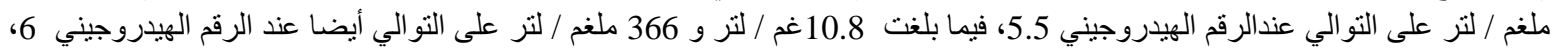

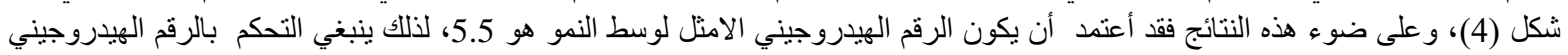

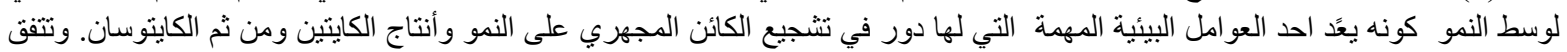

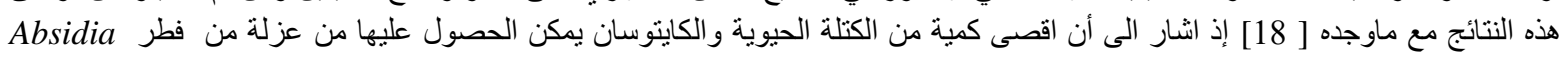
butleri NCIM 977 [17] أن الرقم الهيدروجيني للاوساط التخمرية الصلبة يؤثر بشكل كبير على معدل نمو الفطريات ويتراوح الرقات الرقم الهيدروجيني المثالي لنمو

معظم الفطريات بين 5.9-6.5.

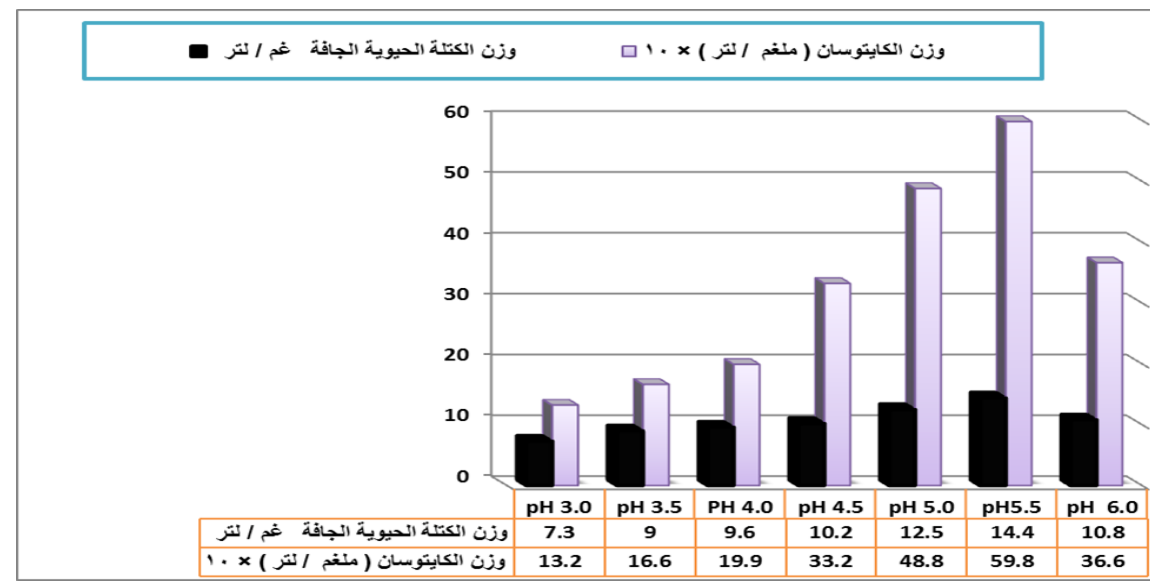

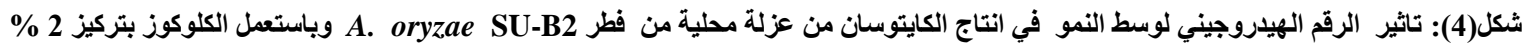

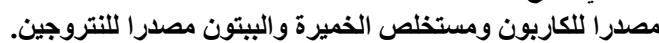


تحديد الحرارة المثلى لانتاج الكايتوسان

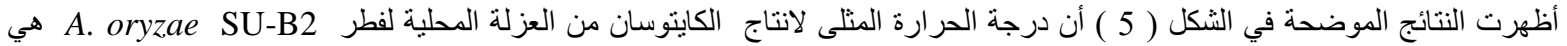

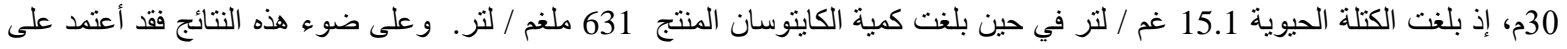

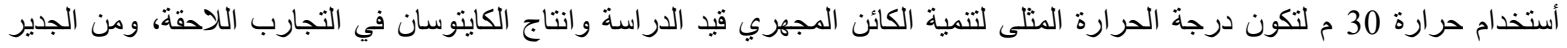

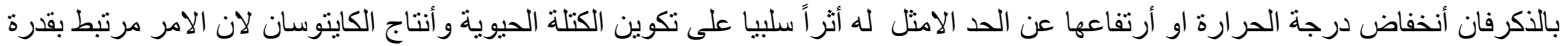

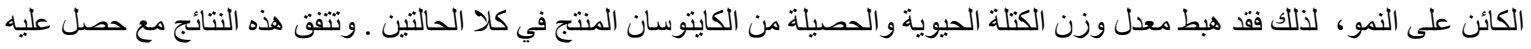

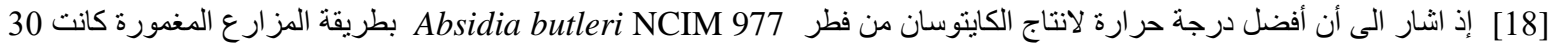

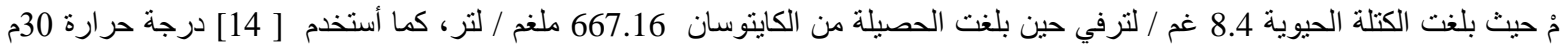

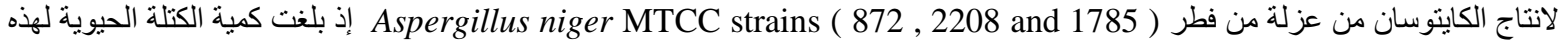

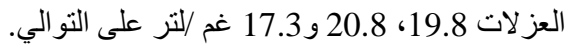

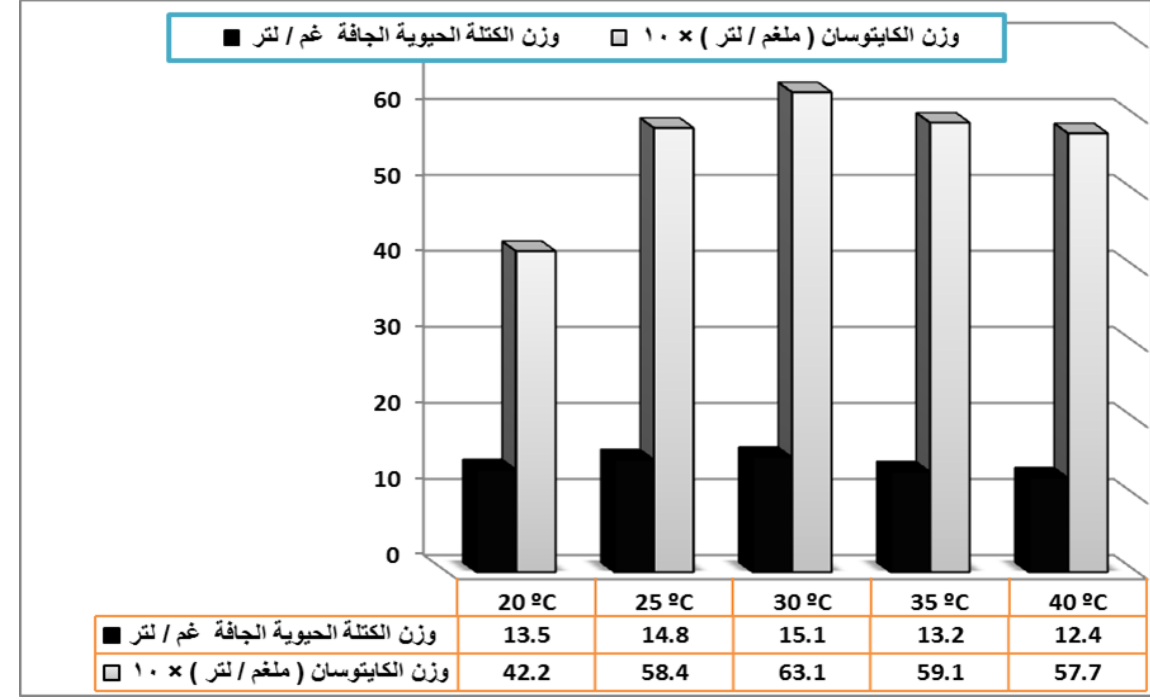

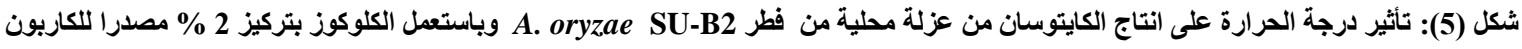
ومستخلص الخميرة واليبتون مصدرا للنتروجين والرقم الهيدروجيني للوسط

تأثير فترة الحضن المثلى لأنتاج الكايتوسان

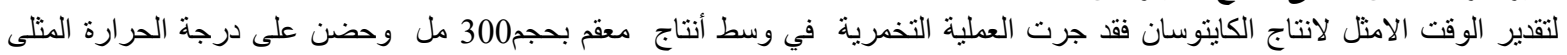

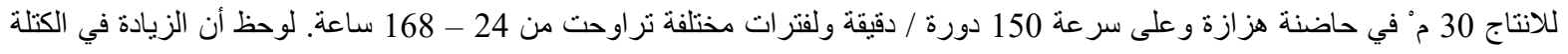

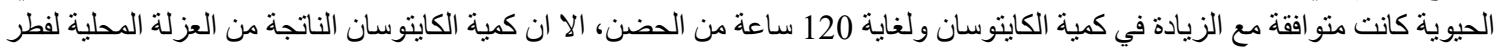
A. oryzae SU-B2

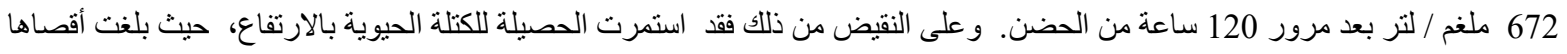

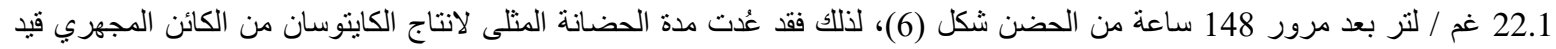

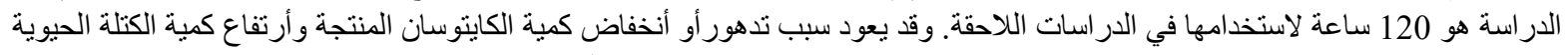

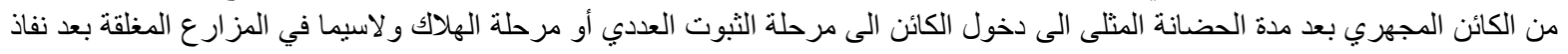

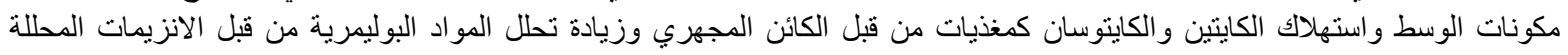

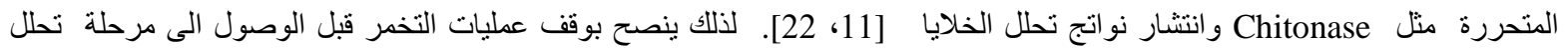

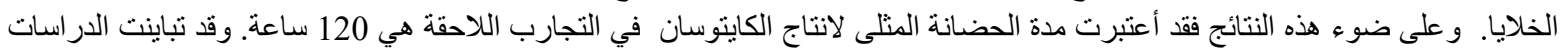
في تحديد مدة الحضانة المتلى لانتاج الكايتو سان حسب نون نوع الكائن المجهري المستخدم في العملية التخمرية. 


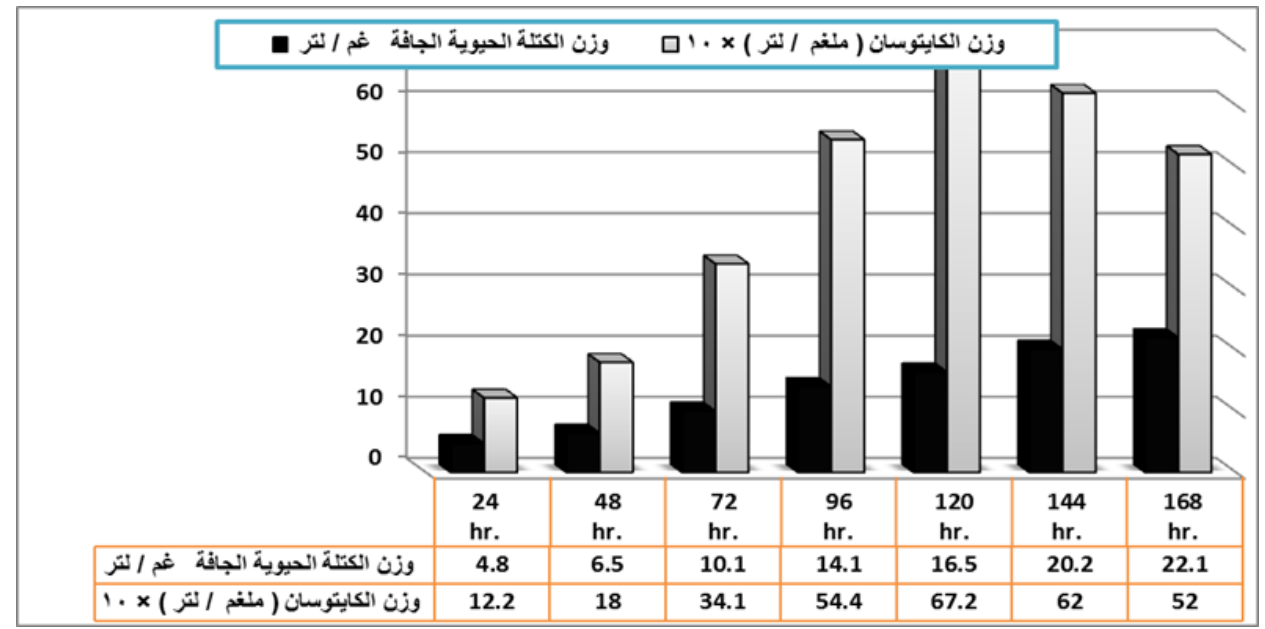

شكل (6): تأثير فترة الحضن على انتاج الكايتوسان من عزلة محلية من عفن A. oryzae SU-B2 وياستعمل الكلوكوز بتركيز 2 \% مونة مصدرا للكاربون

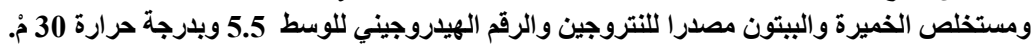

تحديد حجم اللقاح الامثل لانتاج الكايتوسان

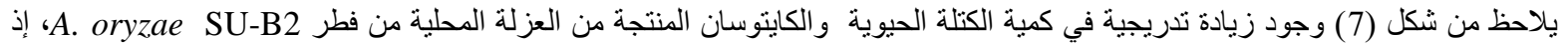

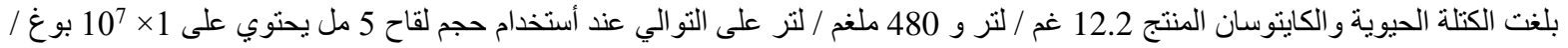

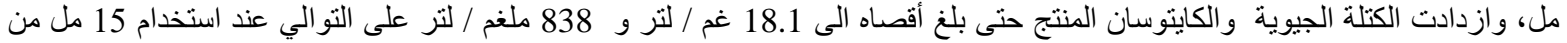

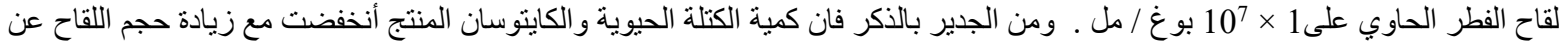

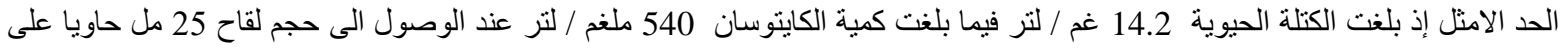

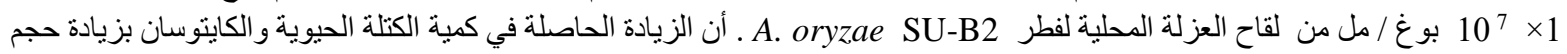

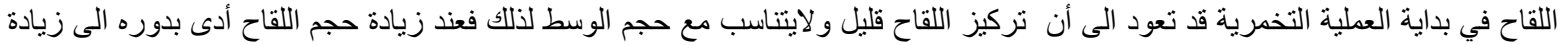

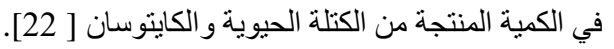

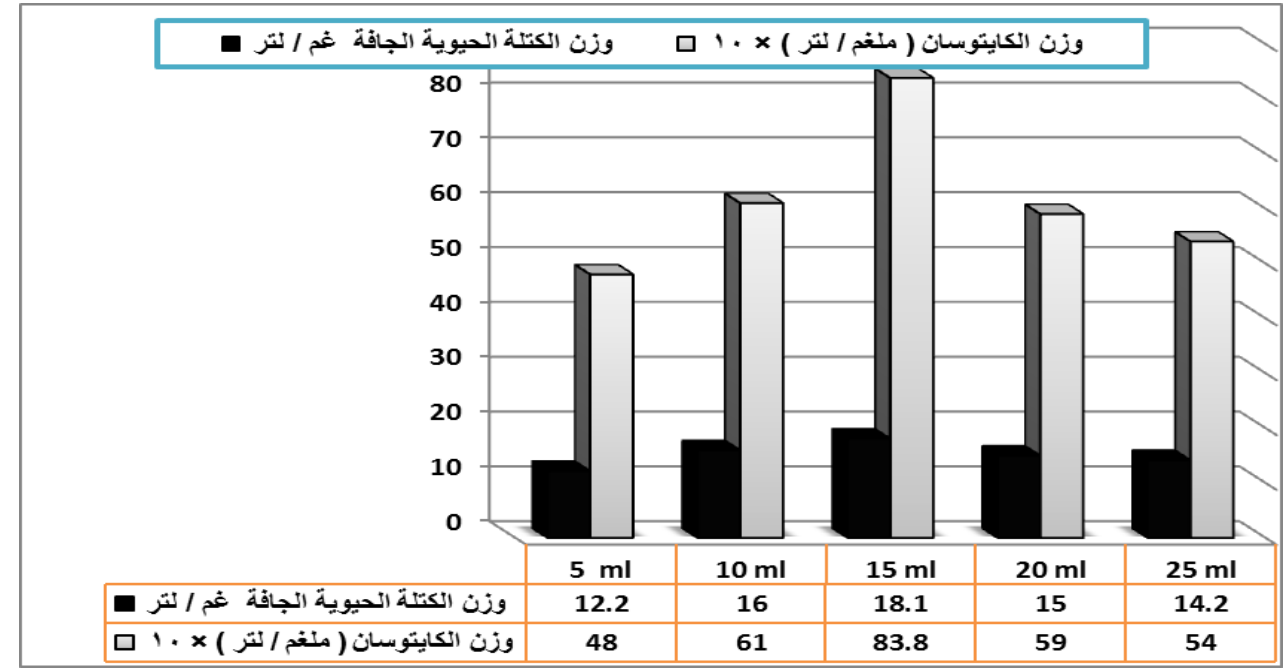

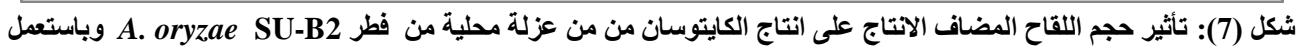

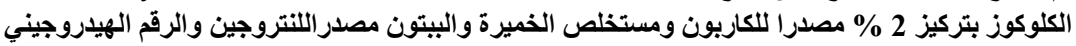
للوسط 25.5 وخلال 120 ساعة من الحضن على درجة 30 مُ.

تأثير درجة التهوية في انتاج الكايتوسان

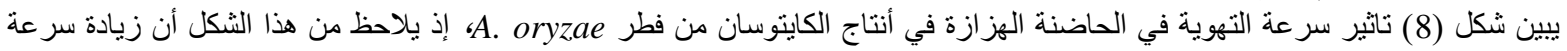

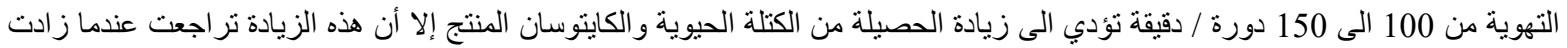

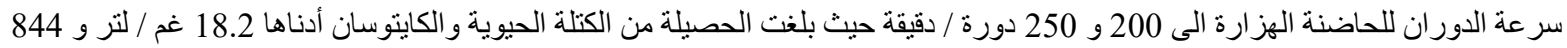

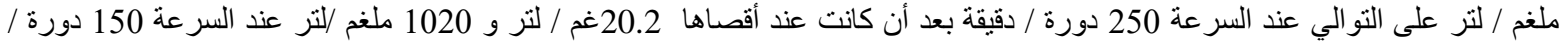

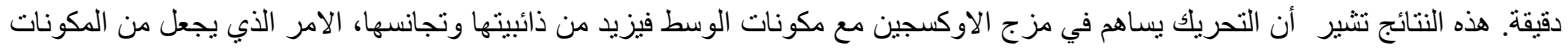

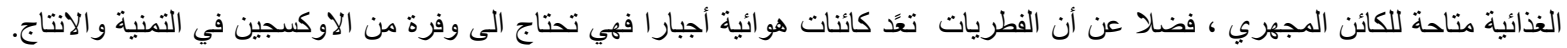

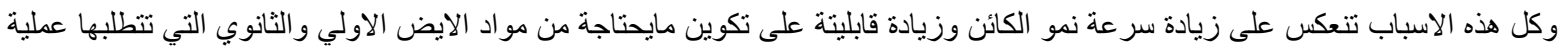

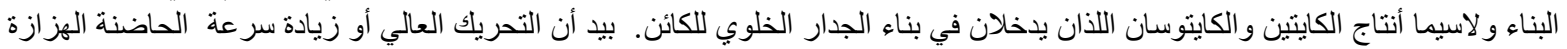




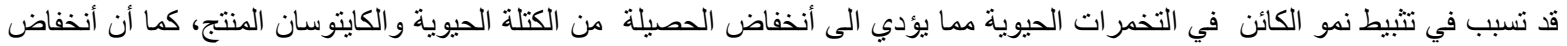

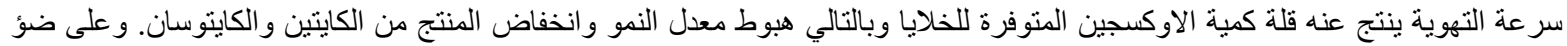

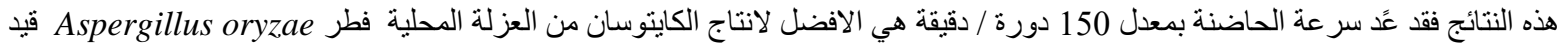

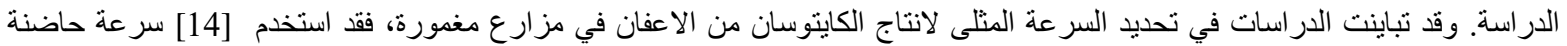
هزارة تعادل 180 دورة / دقيقة لانتاج الكايتوسان من فطر Aspergillu niger، و على خلاف ذلك فقد وجد التان [19] أن أفضل انتاج للكتلة

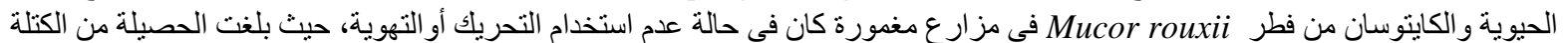

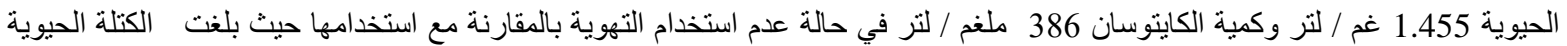
1.355 غم / لتر وكمية الكايتوسان 353 ملغم / لتربة التر

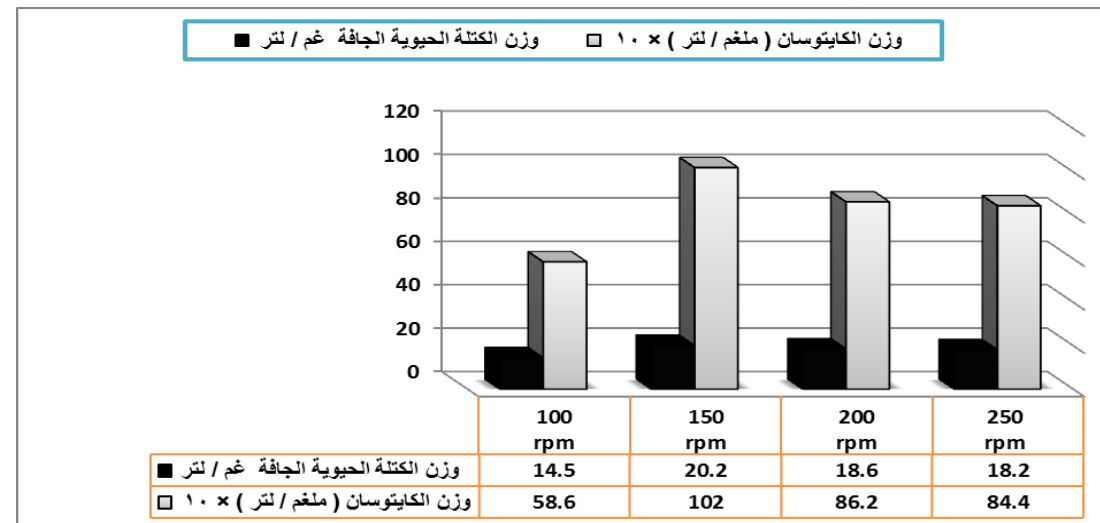

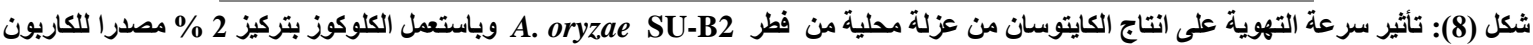

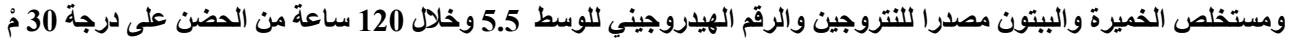

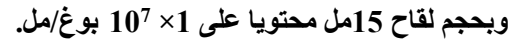

اجمالي الظروف المثلى وكفاءة الانتاج

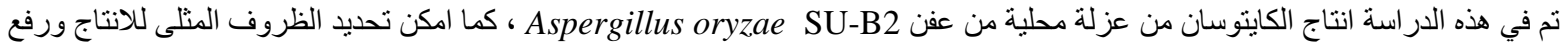
كفاءة الانتاج الى 207.2 \% جدول (1) )

A. oryzae SU-B2 (1) جدول يوضح الظروف المثلى لأنتاج لكايتوسان من الفطر

\begin{tabular}{|c|c|c|c|c|}
\hline $\begin{array}{c}\text { نسبة الزيادة ) } \\
\text { (\% }\end{array}$ & (ملفر/لتزر) & الكتلة الحيوية الجاف & العامل المؤثر الامثل & العامل المؤثر \\
\hline- & 3320 & 9.29 & الكلوكوز & المصدر الكاربوني \\
\hline 39.7 & 4640 & 11.5 & $2 \%$ & تركيزالمصدر الكاربوني \\
\hline 54.2 & 5120 & 13.3 & مستخلص الخميرة + البيتون & المصدر النتروجيني \\
\hline 80.1 & 5980 & 14.4 & 5.5 & الرقم الهيدروجيني \\
\hline 90.0 & 6310 & 15.1 & 30 & درجة الحرارة \\
\hline 102.4 & 6720 & 16.5 & 120 ساعة & فترة الحضن \\
\hline 152.4 & 8380 & 18.1 & 15مل وبمعدل 1 × 710 بوغ / مل & حجم اللقاح \\
\hline 207.2 & 10200 & 20.2 & 150 دورة / دقيقة & سرعة التهوية \\
\hline
\end{tabular}

\section{References}

1. Abd El-Naby, M.S., Helmy, M.H., Mahmoud, M.H. and Eman, I.I. (2014). Production of Some Polysaccharides from Kojic Acid Producing Aspergillus oryzae var. effusus NRC14 Biomass. British Biotechnology Journal. 4(11): 1212-1222.

2. Akila, R.M. (2014). Fermentative production of fungal chitosan, a versatile biopolymer (perspectives and its applications). Advances in Applied Science Research. 5(4): 157-170.

3. Aranaz, I., Mengibar, M., Harris, R., Panos, I., Miralles, B., Acosta, N., Galed, G. and Heras, A. (2009). Functional characterization of chitin and chitosan. Current Chemical Biology. 3: 203-230.

4. Dutta, P.K., Ravikumar, M.N.V. and Dutta, J. (2002). Chitin and Chitosan for versatile applications. JMS Polymer Review. C42: 307.

5. Ghoody, G.W. (1994). Cell walls. In the Growing Fungus p43-51 London, Chapman and Hall.

6. Guha , A.K ., Chatterjee, B.P., Chatterjee, S. and Adhya, M. (2005). Production and Phisico- Chemical Characterization. Process Biochemisty. 40:395-400.

7. Ke-Jin, H., Jin-Lian, H., Kwok-Ping, H. and Kwok-Wing, Y. (2004). Screening of fungal chitosan and chitosanaceous materials. Carbohydrate Polymers. 58: 45-52. 
8. Kumar, A. B. V., Varadaraj, M. C., Gowda, L.R. and Tharanathan, R.N. (2005). Characterization of chitooligosaccharides prepared by chitosanolysis with the aid of papain and Pronase, and their bactericidal action against Bacillus cereus and Escherichia coli. Biochemical journal. 391(2):167-175.

9. Logesh, A.R., Thillaimaharani, K.A, Sharmila, K., Kalaiselvam, M. and Raffi, S.M. (2012). Production of chitosan from endolichenic fungi isolated from mangrove environment and its antagonistic activity. Asian Pacific Journal of Tropical Biomedicine. 2(2): 140-143.

10. Maghsoodi, V., Razavi, J. and Yaghmaei, S. (2009). Solid State fermentation for production of chitosan by Aspergillus niger . International Journal of Engineering- TransactionsA: Basics. 22 (1):1-6.

11. Maghsoodi, V. and Yaghmaei, S. (2010). Comparison of solid substrate and submerged fermentation for chitosan production by Aspergillus niger. Transactions C: Chemistry and Chemical Engineering. 17(2): 153157.

12. Mohammad, A. E., Aroona, C., Eshrat, G., Fathabad and Fereshteh, P. (2013). Preparation of chitossan from Pencillium SPP. and determination of their degree of deacetylation. Indian Journal of Biotechnology. 12:231235.

13. Nadarajah, K., Kader J., Mazmirea, M. and Paul, D.C. (2001). Production of chitosan by fungi. Pakistan Journal of Biological sciences. 4(3): 263-265.

14. Natarajan, K., Riyaz, A. B. and Rengarajan, S. (2011). Production and evaluation of chitosan from Aspergillus niger MTCC Strains, Iranian Journal of Pharmaceutical Research. 10(3):553-558.

15. Nemtsev, S.V., Zueva, O.Y., Khismatullin, M.R., Albulov, A.I., and Varlamov, V.P. ( 2004). Isolation of chitin and chitosan from honey bees. Applied Biochemistry and Microbiology. 40:39-43.

16. Niederhofer, A. and Muller. B.W. (2004). A Method for Direct Preparation of Chitosan with Low Molecular weight from fungi. European Journal of Pharmaceuties and BioPharmaceuties. 57:101-105.

17. Nwe, N. and Stevens, W.F. (2004). Effect of urea on Fungal Chitosan Production in Solid Substrate Fermentation., Process Biochemistry. 39:1639-1642.

18. Pradnya, N.V. and Archana, R.J. (2014). Fermentative production of mycelial chitosan from Zycomycetes: Media Optimization and physico-chemical characterization. Advance in bioscience and biotechnology. (5): 940-956.

19. Sakthivel, L. and Gopal, S. (2013). Studies on Chitosan production from different fungal mycelium. International Journal of Current Biotechnology. 1(1):9-11.

20. Sato, H., Toyoshima, Y., Shintani, T. and Gomi, K. (2011). Identification of potential cell wall component that allow Taka-amylase A adsorption in submerged cultures of Aspergillus oryzae. Applied Microbiology and Biotechnology. 92(5):961-969.

21. Shepherd, R., Reader, S. and Falshaw, A. (1997 ). Chitosan functional properties. Glycoconjugate Journal. 14:535-542.

22. Viccini, G., Mitchell, D.A., Boit, S.D., Gern, J.C., da Rosa, A.S., Costa, R.M., Dalsenter, F.D.H., van Meien, O.F. and Krieger, N. (2001). Analysis of Growth Kinetic Profiles in SSF. Food Technology and biotechnology. 39(4):271-294. 